\title{
Níveis residuais de sais de cura e seu efeito antioxidante em jerked beef
}

\section{Residual levels of curing salts and its antioxidant effect in Jerked beef}

\author{
Elza Youssef Youssef ${ }^{*}$; Carlos Eduardo Rocha Garcia²; Berenice Figueiredo3; \\ Massami Shimokomaki ${ }^{4}$
}

\section{Resumo}

Charque $(\mathrm{CH})$ e Jerked beef (JB) são produtos cárneos salgados que diferenciam entre si pela utilização de sais de cura durante seu processamento. Amostras de $\mathrm{CH}(\mathrm{N}=7)$ e JB $(\mathrm{N}=7)$ adquiridas aleatoriamente em supermercados da região de Londrina foram avaliadas quanto à concentração de nitrato e nitrito de sódio e a sua possível relação com a oxidação lipídica determinada segundo o número de TBARS. As amostras de JB apresentaram concentrações de nitrato entre 27,7 e 83,6 ppm e níveis máximos de 10,0 ppm para nitrito. A rancidez nas amostras de $\mathrm{CH}$ alcançou entre 1,03 a 1,78 mg de TBARS/ $\mathrm{Kg}$ do produto, enquanto no JB estes níveis foram de 0,23 a 1,38 mg TBARS/Kg de amostra. Os resultados demonstraram que as amostras de JB apresentaram concentrações de nitrito inferiores aos limites permitidos pela legislação e a presença dos sais de cura inibiu a oxidação lipídica em níveis até duas vezes menores que nas amostras de $\mathrm{CH}$.

Palavras-chave: Charque. Nitrato de sódio. Nitrito de sódio. Oxidação lipídica. Rancidez.

\begin{abstract}
Charqui meat $(\mathrm{CH})$ and jerked beef $(\mathrm{JB})$ are salted meat products basically differenciate from other by the absence and presence of curing salts, respectively. Seven Charqui meat and Jerked beef samples were obtained at random from Londrina region supermarkets and the possibility of curing salts to act as antioxidants was evaluated by quantitatively measurement of sodium nitrate and nitrite and their relationship its lipid oxidation. Sodium nitrate concentration varied from 27.7 to $83.6 \mathrm{ppm}$ whereas sodium nitrite maximum value was $10.0 \mathrm{ppm}$ in JB samples. The degree of lipid oxidation measured as TBARS in $\mathrm{CH}$ samples ranged from 1.03 to $1.78 \mathrm{mg}$ of TBARS/Kg of sample whereas for JB this variation was from 0.23 to $1.38 \mathrm{mg}$ of TBARS/Kg of sample. Our results demonstrated that JB samples presented lower concentration of sodium nitrite in relation to the limit allowed by the Brazilian legislation and the presence of sodium nitrate and nitrite inhibited notoriously the lipid oxidation in JB samples.
\end{abstract}

Key words: Charqui meat. Sodium nitrate. Sodium nitrite. Lipid oxidation. Rancity.

\footnotetext{
${ }^{1}$ Dra $^{\mathrm{a}}$. Em Ciências de Alimentos - Universidade de São Paulo, USP, Farmacêutico-Bioquímica - Depto de Ciência e Tecnologia de Alimentos. Universidade Estadual de Londrina, UEL. Rod. Celso Garcia Cid. Km 380, PR. 445, CEP 86.051-990, Londrina, PR. E-mail: youssef@uel.br; youssefeyy@hotmail.com

2 Dr. em Ciência dos Alimentos, Depto de Ciência e Tecnologia de Alimentos, Universidade Estadual de Londrina, UEL. Farmacêutico. Endereço atual: Depto de Farmácia, Universidade Federal do Paraná, CEP 80210-170, Curitiba, PR. E-mail: carlos.garcia@ufpr.br

3 Química, bolsista de AT do CNPq. Técnica do Depto de Ciência e Tecnologia de Alimentos. Universidade Estadual de Londrina, UEL.E-mail: berenice@uel.br

4 Dr. em Ciência dos Alimentos. Universidade de São Paulo, Farmacêutico, Depto de Ciência e Tecnologia de Alimentos. Universidade Estadual de Londrina, UEL. Endereço atual: Universidade Tecnológica Federal do Paraná. Av. dos Pioneiros 3636, Londrina, PR. E-mail: mshimo@utfpr.edu.br,mshimo@uel.br

*Autor para correspondência
} 


\section{Introdução}

$\mathrm{O}$ charque $(\mathrm{CH})$ é um produto obtido a partir da tecnologia de obstáculos que se destaca entre os derivados cárneos produzidos no Brasil alcançando grande aceitação pelo consumidor (PINTO et al., 1993, SHIMOKOMAKI et al., 1998). A sua atividade de água entre 0,70 a 0,75 foi determinada pelo nosso grupo (TORRES, et al., 1994) o que o classifica como sendo um produto cárneo de umidade intermediária (LEDWARD,1981, SHIMOKOMAKI et al., 1998). As drásticas condições empregadas durante o processamento do charque são capazes de inibir desenvolvimento de microrganismos patogênicos (LARA et al., 2003), porém, permitem o desenvolvimento de microrganismos fermentativos (PINTO et al., 2002) e não comprometem a qualidade protéica do produto assegurando um elevado valor biológico (GARCIA et al., 2001). Inovações introduzidas no processamento do charque como a adição de nitrato e nitrito de sódio e o acondicionamento em embalagem a vácuo deram origem ao jerked beef (JB) (BISCONTINI; LOPES FILHO; SHIMOKOMAKI, 1992).

A presença de nitrato e nitrito de sódio, além da proteção botulínica (LARA et al., 2000; 2003), melhora a aparência do JB em relação ao $\mathrm{CH}$, conferindo ao mesmo a coloração avermelhada característica dos produtos curados. O efeito antioxidante é outra propriedade do nitrito de sódio em produto cárneos curados proposta originalmente por Pearson, Love e Shorland (1977). Contudo, a utilização de nitrito de sódio deve ser minimizada, pois seu consumo está associado à ocorrência efeitos tóxico (LIJINSKY, 1999). Estudos têm demonstrado que menos de $5 \%$ da ingestão de nitrito e nitrato são derivados de carnes curadas e de resíduos vindos de fontes vegetais (CASSENS 1997a, b; ARCHER, 2002; SEBRANEK; BACUS, 2007). No entanto, Hord, Tang e Bryan (2009) estimou que aproximadamente $80 \%$ das dietas contendo nitratos são derivados do consumo de vegetais.
A utilização de nitrato de sódio nos produtos curados comporta-se como uma reserva repondo os níveis de nitrito de sódio, através da ação de bactérias redutoras prolongando a ação antioxidante dos sais (CASSENS, 1997a).

O cloreto de sódio atua como pró-oxidante dos lipídios e a sua larga utilização no processamento de charque tem papel relevante na promoção da rancificação das gorduras deste produto (TORRES, et al., 1989). Os mecanismos propostos para esclarecer a ação catalítica do $\mathrm{NaCl}$ prevêem que o sal possa deslocar os íons ferro de macromoléculas alterando sua reatividade e distribuição aumentando sua atividade catalítica, propõem-se ainda que o $\mathrm{NaCl}$ possa formar com o ferro um complexo de ação catalítica (DECKER, XU 1998). Nestas condições a temperatura pode catalisar a reação de oxidação lipídica durante o processo de salga (KRISTENSEN; PURSLOW, 2001). Assim, podemos prever que durante o processamento do jerked beef, onde as peças cárneas são expostas ao sol e submetidas a períodos de abafamento, as elevadas temperaturas favorecem a formação do ferro não-heme de propriedades mais catalíticas.

A ingestão de produtos tóxicos originados da oxidação lipídica, como o malonaldeído e os óxidos de colesterol, desperta cada vez mais a preocupação do consumidor devido suas possíveis associações às doenças cardíacas, ao câncer e ao envelhecimento precoce (JIMÉNEZ-COLMENERO; COFRADES; CARBALLO, 2001).

O presente trabalho tem por objetivo determinar os níveis residuais de nitrato e nitrito de sódio e o grau de rancidez, observando a ação antioxidante do nitrito em amostras de Jerked beef tendo como parâmetro amostras de charque.

\section{Material e Métodos}

\section{Material}

Sete amostras de charque e jerked beef, embaladas a vácuo, de 7 marcas distintas foram 
adquiridas em triplicata aleatoriamente no comércio e com vida de prateleira média de 75 dias. As análises foram realizadas em triplicata imediatamente após a homogeneização das amostras em processador de alimentos.

\section{Métodos}

DeterminaçãodonúmerodeTBARS (Substâncias reagentes ao ácido 2-tiobarbitúrico)

A oxidação lipídica foi monitorada pela determinação do número de TBARS de acordo com a técnica de Tarladgis et al. (1960), modificada por Crackel et al. (1988), conforme recomendações de Shahidi et al. (1985).

\section{Quantificação de nitrato e nitrito de sódio}

A determinação de nitrato e nitrito foi realizada segundo a técnica modificada de Follett e Ratcliff (1963) e constou da seguinte metodologia:

\section{Desproteinização das amostras}

Quinze gramas da amostra homogeneizada foram misturadas com 7,5 mL de bórax e 40,0 $\mathrm{mL}$ de água destilada e aquecida a $70^{\circ} \mathrm{C}$ por 15 minutos com freqüente agitação. Posteriormente, as amostras foram resfriadas à temperatura ambiente sendo em seguida, adicionados $3,0 \mathrm{~mL}$ de solução de ferrocianeto de potássio e $3,0 \mathrm{~mL}$ de acetato de zinco e deixadas em repouso por 30 minutos sob a proteção da luz. Transferiu-se a amostra para balão de $100,0 \mathrm{~mL}$ e completou-se o volume com água destilada. As amostras foram filtradas em papel de filtro livre de nitrato e nitrito de sódio.

\section{Determinação de nitrito}

Quinze $\mathrm{mL}$ da amostra desproteinizada foram transferidas para um balão volumétrico de $50,0 \mathrm{~mL}$ juntamente com 5,0 mL de solução tampão e 10,0 mL de solução de alfa-naftol. Os balões foram incubados em banho-maria a $25-30^{\circ} \mathrm{C}$ por 30 minutos para o desenvolvimento de cor. A amostra foi resfriada à temperatura ambiente e efetuou-se a leitura a 474 nm. Os cálculos da quantidade de nitrito de sódio presente nas amostras foram determinados através de uma curva padrão previamente estabelecida.

\section{Determinação do nitrato de sódio}

\section{Preparo da coluna de cádmio}

Bastões de zinco foram colocados em béquer contendo $100 \mathrm{~mL}$ de solução de sulfato de cádmio a 20\%. Após 3 horas, removeu-se o depósito esponjoso formado colocando-o em um béquer contendo água destilada. Transferiu-se o cádmio com aproximadamente $200 \mathrm{~mL}$ de água destilada para um liqüidificador e triturou-se por 5 minutos. Posteriormente, as amostras foram passadas em tamis de 20-40 mesh e transferidas para uma coluna de vidro de $15 \mathrm{~mm}$ de diâmetro interno e $100 \mathrm{~mm}$ de altura. Antes da determinação de nitrato de sódio, lavou-se a coluna com 25,0 mL de $\mathrm{HCl} 0,1 \mathrm{~N}$, e em seguida com 50,0 mL de água destilada e finalmente com 25,0 mL de solução tampão diluída 1:9 com água destilada.

\section{Quantificação do nitrato de sódio}

Alíquotas de 20,0 mL da solução desproteinizada da amostra foram transferidas para um béquer de 100,0 mL e adicionou-se solução tampão até obterse uma solução transparente. Colocou-se o conteúdo do béquer na coluna redutora, a uma velocidade de $5,0 \mathrm{~mL} / \mathrm{min}$ rejeitando as 10 primeiras gotas. Lavou-se o béquer com água destilada passando através da coluna até recolher-se 100,0 mL do eluato em um balão volumétrico de 100,0 mL. Posteriormente, $10,0 \mathrm{~mL}$ do eluato foram pipetados e transferidos juntamente com 5,0 mL de solução tampão e 10,0 mL de alfa-naftol para um balão volumétrico de $25,0 \mathrm{~mL}$. A solução foi incubada em banho-maria à $25-35^{\circ} \mathrm{C}$ por 30 minutos para o 
desenvolvimento de cor. A amostra foi esfriada à temperatura ambiente e procedeu-se a leitura a 474 nm. O cálculo da quantidade de nitrato de sódio na amostra foi determinado pela conversão à nitrito de sódio, quantificado através de uma curva padrão previamente estabelecida.

\section{Resultados e Discussão}

Os resultados relativos ao número de substâncias reagentes ao ácido 2-tiobarbitúrico foram obtidos a partir da multiplicação da absorbância encontrada pelo fator 5,67 determinado experimentalmente. A recuperação obtida durante a destilação foi de $90 \%$, relativamente superior ao valor de $68 \%$ encontrado originalmente por Tarladgis et al. (1960).

A Tabela 1 apresenta a intensidade da oxidação lipídica avaliada segundo os valores de TBARS do charque e Jerked beef. Os valores médios de TBARS em charque variaram de 1,03 a $1,78 \mathrm{mg}$ TBARS $/ \mathrm{kg}$ de amostra e JB de 0,23 a 1,38 mg TBARS/kg de

Tabela 1. Valores da determinação de nitrato, nitrito de sódio (ppm) e TBARS ( $\mathrm{mg} / \mathrm{kg})$ em Jerked Beef e charque $(\mathrm{CH})$.

\begin{tabular}{lcrcrc}
\hline & \multicolumn{2}{c}{ Jerked beef } & \multicolumn{2}{c}{ Charque } \\
\hline Amostras & Nitrito de sódio & Nitrato de sódio & TBARS & Amostra & TBARS \\
\hline JB 1 & -- & $27,76( \pm 0,25)$ & $0,74^{\mathrm{f}}( \pm 0,028)$ & $\mathrm{CH} 1$ & $1,15^{\mathrm{d}}( \pm 0,033)$ \\
JB 2 & -- & $56,62( \pm 0,36)$ & $0,93^{\mathrm{e}}( \pm 0,043)$ & $\mathrm{CH} 2$ & $1,15^{\mathrm{d}}( \pm 0,009)$ \\
JB 3 & $2,90( \pm 0,01)$ & $38,68( \pm 0,14)$ & $1,38^{\mathrm{c}}( \pm 0,037)$ & $\mathrm{CH} 3$ & $1,03^{\mathrm{e}}( \pm 0,015)$ \\
JB 4 & -- & $74,40( \pm 0,44)$ & $0,59^{\mathrm{g}}( \pm 0,026)$ & $\mathrm{CH} 4$ & $1,25^{\mathrm{d}}( \pm 0,059)$ \\
JB5 & $10,0( \pm 0,06)$ & $83,61( \pm 0,38)$ & $0,36^{\mathrm{h}}( \pm 0,010)$ & $\mathrm{CH} 5$ & $1,40^{\mathrm{c}}( \pm 0,051)$ \\
JB 6 & $2,92( \pm 0,05)$ & $60,76( \pm 0,26)$ & $0,36^{\mathrm{h}}( \pm 0,015)$ & $\mathrm{CH} 6$ & $1,78^{\mathrm{a}}( \pm 0,014)$ \\
JB 7 & $4,04( \pm 0,002)$ & $72,77( \pm 0,44)$ & $0,23^{\mathrm{i}}( \pm 0,007)$ & $\mathrm{CH}$ & $1,52^{\mathrm{b}}( \pm 0,051)$ \\
\hline
\end{tabular}

Os valores representam a média de três repetições analisados em triplicatas.

(dp) desvio padrão das médias. (--) traços

Letras diferentes diferem significativamente a nível de 5\% pelo teste de Tukey.

Os resultados obtidos neste estudo demonstraram experimentalmente a ação antioxidante dos sais de nitrato e nitrito de sódio no jerked beef, resultando em número de TBARS até duas vezes menores no Jerked beefem relação ao charque. Torres et al. (1989) aplicando nitrato de sódio às amostras de charque não encontrou efeito antioxidante, sugerindo que a alta concentração de sal provavelmente impediu o crescimento de bactérias capazes de reduzir o nitrato a nitrito, desde que somente o nitrito apresenta propriedades antioxidantes (CHEN et al., 1984).

Na Tabela 1 é possível verificar que as amostras com maiores concentrações (valores absolutos) de nitrato apresentam menores valores de TBARS. Os resultados demonstraram que a associação entre nitrato e nitrito de sódio exerceu ação antioxidante no JB, sugerindo que os microrganismos foram capazes de reduzir o nitrato a nitrito.

A atividade antioxidante do nitrito está associada à sua ação quelante sobre os metais catalíticos da oxidação, à capacidade de combinar-se e estabilizar as insaturações dos ácidos graxos e a propriedade deste sal associar-se aos íons ferro, presentes em macromoléculas, impedindo que atuem como catalisadores da oxidação lipídica (ARENDT; SKIBSTED; ANDERSEN, 1997). 
A Tabela 1 apresenta ainda os valores obtidos na determinação de nitrato e nitrito. De acordo com a legislação vigente, em JB é permitido um resíduo final de $50 \mathrm{ppm}$ de nitrito no produto (BRASIL, 2000). As amostras analisadas apresentavam concentrações de nitrito inferior aos valores observados para nitrato. A metodologia utilizada permitiu detectar nas amostras JB 1, JB 2, e JB 4 somente traços de nitrito e nas demais amostras os valores máximos encontrados foram de $10 \mathrm{ppm}$. Podemos verificar que as amostras analisadas apresentavam concentrações de nitrito abaixo do valor máximo permitido segundo a legislação vigente (BRASIL, 2000).

\section{Conclusões}

As concentrações encontradas para nitrito de sódio nas amostras de Jerked beef foram inferiores ao limites propostos pela legislação em vigor e os valores absolutos de TBARS foram até duas vezes menores que os obtidos para o charque, indicando a efetiva ação antioxidante dos sais de cura.

\section{Referências}

ARCHER, D. L. Evidence that ingested nitrate and nitrite are beneficial to health. J. Food Protect., Des Moines, v. 65, n. 5, p. 872-875, 2002.

ARENDT, B.; SKIBSTED, L. H.; ANDERSEN, H. J. Antioxidative activity of nitrite in metmyoglobin induced lipid peroxidation. Z Lebensm Unters Forsch A, Frankfurt, v. 204, n. 1, p. 7 -12, 1997.

BISCONTINI, T. M. B.; LOPES FILHO, A.; SHIMOKOMAKI, M. Jerked beef: uma evolução tecnológica do charque. Revista Nacional de Carne, São Paulo, v. 16, n. 183, p. 43, 1992.

BRASIL, Ministério da Agricultura. Departamento Nacional de Inspeção de Produtos de origem animal. Circular $n^{\circ}$ 16/DICAR de 24/01/2000. Brasília, 2000.

CASSENS, R. G. Composition and safety of cured meats in the USA. Food Chemistry, Barking, v. 59, n. 4, p. 561$566,1997 \mathrm{a}$.

Residual nitrite in cured meat. Food Technol., Chicago, v. 51, n.2, p. 53-55. 1997 b.
CHEN, C. C.; PEARSON, A. M.; GRAU, J. L.; FOOLADI, M. H.; KU, P. K. Some factors influencing the nonheme iron content of meat and its implications in oxidation. Journal of Food Science, Chicago, v. 49, n. 2, p. 581-584, 1984.

CRACKEL, R. L.; GRAY, J. I.; PEARSON, A. M.; BOOREN, A. M.; CUCKLEY, O. J. Some further observation on the TBA test as an index of lipid in meats. Food Chemistry, Kindlington, v. 28, n. 3, p. 187-196, 1988.

DECKER, E. A.; XU, Z. Minimizing rancidity in muscle foods. Food Technology, Chicago, v. 52, n. 10, p. 54-59, 1998.

FOLLETT, M. J.; RATCLIFF, P. W. Determination of nitrite and nitrate in meat products. Journal of Science Food Agriculture, London, v. 14, n. 3, p. 138-144, 1963.

GARCIA, F. A.; MIZUBUTI, I.; KANASHIRO, M.; SHIMOKOMAKI, M. Intermediate moisture meat product. biological evaluation of charqui protein quality. Food Chemistry, Exeter, v. 75, n. 4, p. 19-23, 2001.

HORD, N. G.; TANG, Y.; BRYAN, N. S. Food sources of nitrates and nitrites: the physiologic context for potential health benefits. Am. J. Clin. Nutr., Houston, v. 90, n. 1, p. 1-10. 2009.

JIMÉNEZ-COLMENERO， F.; COFRADES， J.; CARBALLO, L. C. Healthier meat and meat products: their role as functional foods. Meat Science, Barking, v. 59, n. 1, p. 5-3, 2001.

KRISTENSEN, L.; PURSLOW, P. P. The effect of processing temperature and addition of mono- and divalent salts on the heme-nonheme-iron ratio in meat. Food Chemistry, Exeter, v. 73, n. 4, p. 433-439, 2001.

LARA, J. A. F.; DUTRA, I. S.; PINTO, M. F.; SHIMOKOMAKI, M. Intermediate moisture meat product. Evaluation of botulinal toxin production in charqui meat. In: 46 Th International Congress Of Meat Science And Technology, Buenos Aires, v. 2, p. 738-739. 2000.

LARA, J. A. F.; SENIGALIA, S. W. B.; OLIVEIRA, T. C. R. M.; DUTRA, I. S.; PINTO, M. F.; SHIMOKOMAKI, M. Evaluation of survival of Staphylococcus aureus and Clostridium botulinum in chaqui meats. Meat Science, Exeter, v. 65, n. 1, p. 609-613, 2003.

LEDWARD, P. Intermediate moisture meats. In: LAWRIE, R. (Ed.). Developments in meat science. London: Applied Science, 1981. v. 2, p. 159-194.

LIJINSKY, W. N.-Nitroso compounds in the diet. Mutation Res.-Genet. Toxicol. Environ. Mutagen, Amsterdam, Netherlands, v. 443, n.1/2, p. 129-138, 1999. 
PEARSON, A. M.; LOVE, J. D.; SHORLAND, F. B. Warmed-over flavor in meat, poultry and fish. Advances in Food Research, São Diego, v. 23, n. 1, p. 1-74, 1977.

PINTO, M. F.; PONSANO, E. H. G.; FRANCO, B. D. G. M.; SHIMOKOMAKI, M. Charque e sucedâneos são produtos cárneos obtidos por processos combinados (Hurdle Technology). Revista Nacional da Carne, São Paulo, v. 17, n. 195, p. 66-68, 1993.

PINTO, M. F.; PONZANO, E. H. G.; FRANCO, B.D.G.M.; SHIMOKOMAKI，M. Charqui meats as fermented meat products: role of bacteria for some sensorial properties development. Meat Science, Barking, v. 61, n. 2, p. 187-191, 2002.

SEBRANEK, J. G.; BACUS, J. Cured meat products without direct addition of nitrate and nitrite: what are the issues? Meat Sci., Barking, v. 77, n. 1, p. 136-147. 2007.

SHAHIDI, E.; RUBIN, L. J.; DIOSADY, L. L.; WOOD, D. F. Effect of sulphanilamide on the TBA values of cured meats. Journal of Food Science, Chicago, v. 50, n. 1, p. 274-275, 1985.

SHIMOKOMAKI, M.; FRANCO, B. D. G. M.; BISCONTINI, T. M. B.; PINTO, M. F.; TERRA, N. N.; ZORN, T. M. T. Charqui meats are hurdle technology meat products. Food Reviews International, New York, v. 14, n. 4, p. 339-349, 1998.

TARLADGIS, B. G.; WALTS, B. M.; YOUNATHAN, M. T.; DUCAN J. R.L. R. A destillation method for quantitative determination of malonaldehyde in rancid foods. Journal America Oil Chemistry Society, Champaign, v. 37, n. 1, p. 44-48, 1960.

TORRES, E. A. F. S.; PEARSON, A. M.; GRAY, J. I.; KU, P. K.; SHIMOKOMAKI, M. Lipid oxidation in charqui (salted and dried beef). Food Chemistry, Exeter, v. 32, n. 4, p. 257-268, 1989.

TORRES, E. A. F. S.; SHIMOKOMAKI, M.; FRANCO, B. D. G. M.; LANDGRAF, M.; CARVALHO JUNIOR, B. C. C.; SANTOS, J. C. Parameters determining the quality of charqui, an intermediate moisture meat product. Meat Sci., Barking, v. 38, n. 2, p. 229-234, 1994. 